\title{
Open access at Nature Methods
}

\author{
New year, big changes: Nature Methods now offers authors the ability to publish research papers on an open access \\ basis, including via a Guided Open Access pilot. Here's how it works.
}

Fisis rom January 2021 on, we are pleased to offer authors of primary research papers an expanded array of publishing options, including open access (OA). Nature and the Nature Research journals, including Nature Methods, have become 'transformative journals', meaning that while we still offer traditional subscription-based publication, we aim to increase the number of OA papers we publish each year, with the ultimate goal of becoming a fully OA journal.

This news, communicated in November, was not entirely warmly embraced by the research community, mainly due to the cost $(€ 9,500$ per article, or about $\$ 11,500)$ of publishing OA at Nature and the Nature Research journals. This article processing charge reflects some realities of publishing at a Nature journal: because we publish few papers as compared to the number of submissions, OA costs must be shouldered by few authors. Only a small percentage of manuscripts are peer reviewed (in 2020 at Nature Methods, this number was about $10 \%)$, meaning that editors spend a large portion of their time evaluating submissions that are rejected. Manuscripts that are peer reviewed, however, get a substantial amount of editorial attention and guidance throughout the publication process, which many authors find highly valuable. And a team of additional staff members, including copy editors, production editors and art editors, all handle the final product, ensuring its highest possible quality.

We understand that this article processing charge may be a stretch for many labs. However, authors belonging to institutions with 'transformative agreements' with Nature and the Nature Research journals will be able to publish manuscripts $\mathrm{OA}$ at no extra cost. We are also excited to offer a lower cost possibility for publishing OA in Nature Methods, with our Guided Open Access pilot program.

Authors who submit primary research manuscripts via our web portal will be offered the Guided Open Access option, in addition to the regular option (where authors may choose at a later stage whether to publish OA or by the traditional subscription route). Authors selecting this option will have their manuscript concurrently considered for publication at Nature Methods, Nature Communications and Communications Biology. Editors from all three journals will discuss the paper, and if the manuscript meets the editorial criteria for potential publication in at least one of the journals, it will be sent out for peer review. At this stage, authors will need to pay an editorial assessment charge of $€ 2,190$ (note that this fee applies only to papers that are being sent out for peer review). In exchange for this service fee, authors will receive an Editorial Assessment Report. This report will include the peer reviewer comments, annotated by the editors with detailed feedback about how to best address the points raised in order to satisfy the requirements for publication in one or more of the three journals. The report will also include detailed guidance to help authors conform to best practices for transparency and reproducibility.

Authors will receive this detailed report no matter whether their manuscript is invited for revision or whether it is declined following peer review. If the manuscript is rejected, or if authors choose to withdraw and submit it elsewhere, their reviewer reports will be portable, including to other publishers. Authors whose manuscripts are eventually accepted into either Nature Methods or Nature Communications will be asked to pay a top-up fee of $€ 2,600$; manuscripts accepted into Communications Biology will have a top-up fee of $€ 800$. The total fee for publishing in Nature Methods via the Guided Open Access route is therefore $€ 4,790$, about half the standard OA fee.

We will apply the very same editorial standards at Nature Methods no matter which publishing option authors choose. But, by submitting via the Guided Open Access route, authors may save time by having their paper considered concurrently by three different journals.

For the time being, unless authors' funders demand OA publication, authors may also continue to choose the traditional subscription route. Though in such a case the journal version of the paper will not be OA, Nature and the Nature Research journals support sharing pre-review manuscript versions via preprint servers, archiving of pre-acceptance manuscript versions 6 months after publication in public repositories, and unrestricted sharing of the full journal version via read-only SharedIt links.

Many researchers have passionate feelings about OA. However, OA is really just one aspect of the broader ideals of open science, which we fully embrace. One of our main roles as editors is to ensure that the research papers we publish conform to best practices in open science. This includes careful editing of papers to ensure that the methods are well described, data availability is detailed, code is verified to run and is shared if central to the paper, and unique materials are fully described and characterized. We also work to ensure that papers meet the highest standards of reporting to achieve reproducibility, that the statistics used are appropriate, and that authors are transparent about funding sources and conflicts of interest.

Open science also means acknowledging the people involved in shaping a paper. Nature Methods already names the editor responsible for handling a published paper. We also offer peer reviewers the option to sign their reports and to be acknowledged by name in published papers. We will also soon be offering all of our authors the option of publishing the reviewer reports alongside their papers.

Nature Methods is editorially independent of our publisher, Springer Nature; editors are not involved in making decisions regarding publishing models. However, we believe that providing new OA publishing options for our authors is a positive development for the communities we serve. We advise authors to choose the publishing option that best suits their funders' demands, their willingness to allow our editors to guide the paper to the journal that is the best fit (if selecting the Guided Open Access route) and their own personal opinions about OA.

As always, we are happy to answer questions and take feedback on our editorial processes.

Published online: 4 February 2021 https://doi.org/10.1038/s41592-021-01073-y 